\title{
RF Tomography in Free Space: Experimental Validation of the Forward Model and an Inversion Algorithm Based on the Algebraic Reconstruction Technique
}

\author{
V. Picco, T. Negishi, S. Nishikata, D. Spitzer, and D. Erricolo \\ Department of Electrical and Computer Engineering, University of Illinois at Chicago, Chicago, IL 60607, USA \\ Correspondence should be addressed to D. Erricolo; derricl@uic.edu
}

Received 28 March 2013; Accepted 22 May 2013

Academic Editor: Francesco Soldovieri

Copyright (c) 2013 V. Picco et al. This is an open access article distributed under the Creative Commons Attribution License, which permits unrestricted use, distribution, and reproduction in any medium, provided the original work is properly cited.

Radio-frequency tomography was originally proposed to image underground cavities. Its flexible forward model can be used in free-space by choosing an appropriate dyadic Green's function and can be translated in the microwave domain. Experimental data are used to validate a novel inversion scheme, based on the algebraic reconstruction technique. The proposed method is improved by introducing physical bounds on the solution returned. As a result, the images of the dielectric permittivity profiles obtained are superior in quality to the ones obtained using classical regularization approaches such as the truncated singular value decomposition. The results from three experimental case studies are presented and discussed.

\section{Introduction}

The experimental validation of the forward model and an inversion scheme of RF Tomography are considered.

$\mathrm{RF}$ tomography was originally proposed by Wicks [1] to image the presence of underground cavities or tunnels. RF tomography consists of a set of transmitters that illuminate the region of interest. If an object is present, part of the illumination is scattered towards the surface, where it is measured by a set of receivers. Then, the measurements are processed to produce an image of the underground. The theoretical formulation of RF tomography [2-6] is based on a forward model to account for the propagation of the electromagnetic field from the transmitters to the scatterer and back to the receivers, and on an inversion method to produce the images.

The forward model formulation of RF tomography is based on a classical volume integral equation approach $[7,8]$ and uses the Born approximation. Although it had been originally developed for the application of underground imaging, operating at high frequency $(3 \mathrm{MHz}-30 \mathrm{MHz})$ or below, RF tomography can be applied to detect the aboveground and below-ground targets [9], and its frequency of operation can be shifted into the microwave domain [10].
Regarding the inversion procedures, traditionally $\mathrm{RF}$ tomography has employed direct (noniterative) regularized inversion techniques such as the truncated singular value Decomposition (TSVD) or the Tikhonov regularization. These methods tackle the problem of illconditioning but often fail to produce highly resolved, sharp images.

Some preliminary measurements aimed to validate RF Tomography [11] (operating at $400-1000 \mathrm{MHz}$ ) highlighted difficulties in taking measurements in an actual open field. Therefore, we chose to consider laboratory measurements in the microwave frequency domain.

Three fundamental requirements dictated the choice of these laboratory experiments. First, the goal of reconstruction: in RF tomography the goal is to identify the location of a target, which does not require the accurate reconstruction of its electrical properties. The target is assumed to be an isolated anomaly, with sharp contrast with respect to the host medium, and with simple shape. Second, the measurement conditions: RF tomography had been originally developed for reasons of detection and surveillance in defense systems, thus dealing with possible hostile environments. Successful imaging needs to be achieved with a limited number of sensors and must be robust against missing or broken antennas. Third, the time required to collect and process data to obtain images: 
short times are critical in RF Tomography because the goal is to obtain real-time information about the target position and, possibly, to perform tracking. It is also imperative to retrieve and process information quickly because when sensors are deployed in a hostile environment, they may no longer be available at some point.

Therefore, there emerges the need to implement a quick inversion algorithm, which would improve the quality of the images obtained using direct regularization methods. While able to operate with a limited number of measurements, a quick inversion possibly sacrifices the exact quantitative reconstruction of the dielectric permittivity of the target. Hence, these requirements render the experiments for RF Tomography described in this paper fundamentally different from experiments for Microwave Tomography systems, generally developed for medical applications, such as those described in [12-14].

There are three contributions in this paper. First, we detail the characteristics of our measurement system; second, we describe an inversion algorithm based on the Algebraic Reconstruction Technique that allows us to introduce physical constraints and to address the three requirements detailed previously; third, we use the measurement system to verify the validity of the RF Tomography forward model and to assess the quality of reconstruction using our inversion method.

\section{Materials and Methods}

2.1. Forward Model. The goal of the forward model is to link the quantity being measured (the scattered electric field) with the quantity being reconstructed. In RF Tomography we consider as contrast function the quantity $\varepsilon_{\delta}$, which represents the difference between the unknown complex permittivity of the target and the known complex permittivity of the medium where the target is embedded. The formulation is considered valid inside a certain integration domain $D$.

As a result, the vectorial forward model for the scattered field is [2]

$$
E^{s}(\mathbf{t}, \mathbf{r})=Q \int_{D} \mathbf{a}_{m}^{r} \cdot \underline{\underline{G}}\left(\mathbf{r}, \mathbf{r}^{\prime}\right) \cdot \underline{\underline{G}}\left(\mathbf{r}^{\prime}, \mathbf{t}\right) \cdot \mathbf{a}_{n}^{t} \varepsilon_{\delta}\left(\mathbf{r}^{\prime}\right) d \mathbf{r}^{\prime}
$$

where $\mathbf{a}_{m}^{r}$ and $\mathbf{a}_{n}^{t}$ are unit vectors representing the orientation of, respectively, receiving and transmitting ideal Hertzian dipoles ( $t$ and $r$ are used to identify transmitting and receiving antennas more clearly). $Q$ is a constant which depends on the characteristics of the antennas used, such as their effective height; for example, using short dipoles of length $\Delta l$, driven with a current of magnitude $I$ at frequency $f, Q=j 2 \pi f \mu_{0} \Delta l I$. The dyadic Green's function $\underline{\underline{G}}$ embeds all the information concerning the space where the system is operating. The frequency of operation affects the Green's function by means of the wave number $k_{0}$. Finally, the contrast function $\varepsilon_{\delta}\left(\mathbf{r}^{\prime}\right)$ is the object of the reconstruction and it is defined as,

$$
\varepsilon_{\delta}\left(\mathbf{r}^{\prime}\right)=\varepsilon_{r}\left(\mathbf{r}^{\prime}\right)-\varepsilon_{D}-j \frac{\sigma\left(\mathbf{r}^{\prime}\right)-\sigma_{D}}{2 \pi f \varepsilon_{0}},
$$

where $\varepsilon_{D}$ and $\sigma_{D}$ identify the dielectric permittivity and conductivity of the host medium, respectively.

The model of (1) is linear: it is derived using the Born approximation, which is valid for weak scatterers and ignores the effect of multiple reflections.

It is possible to translate RF Tomography to the microwave domain and to a free-space scenario by choosing [7]

$$
\underline{\underline{\mathbf{G}}}\left(\mathbf{r}, \mathbf{r}^{\prime}\right)=\left[\underline{\underline{\mathbf{I}}}+\frac{\nabla \nabla}{k_{0}^{2}}\right] \frac{e^{j k_{0}\left|\mathbf{r}-\mathbf{r}^{\prime}\right|}}{4 \pi\left|\mathbf{r}-\mathbf{r}^{\prime}\right|},
$$

using the desired frequency. Equation (1) can then be discretized and recast in matrix form as $\mathrm{E}^{S}=\mathbf{L} \cdot \boldsymbol{\varepsilon}_{\delta}$.

2.2. Inversion. To recover $\boldsymbol{\varepsilon}_{\delta}$ we developed an algorithm based on the Algebraic Reconstruction Technique (ART). ART, also known as Kaczmarz method, had been originally developed in the 1930s to iteratively solve linear systems of equations and had been rediscovered in the 1970s to create images in tomographic applications. The algorithm is loosely based on the Moore-Penrose pseudoinverse, in the sense that it follows the pseudoinverse formulation so as to operate on one row of the matrix at a time:

$$
\mathbf{L}_{i}^{\dagger, \mathrm{ART}}=\mathbf{L}_{i}^{H}\left(\mathbf{L}_{i} \mathbf{L}_{i}^{H}\right)^{-1}=\frac{\mathbf{L}_{i}^{H}}{\left\|\mathbf{L}_{i}\right\|_{2}^{2}},
$$

where $\mathbf{L}_{i}$ is a vector corresponding to the $i$ th row of $\mathbf{L}$. Therefore, (4) involves the dot product of two vectors which by definition returns a scalar number. The inverse operation is thus simplified because it is not a matrix inversion, but simply the reciprocal of a real positive scalar, hence the computational advantage. The "row-wise inverse" obtained in (4) is then used to compute the solution to the inverse problem in an iterative algorithm. Starting from a guessed solution $\boldsymbol{\varepsilon}_{\delta}^{(0)}$ (usually the null vector), at every iteration $(n)$ the algorithm computes

$$
\boldsymbol{\varepsilon}_{\delta}^{(n+1)}=\boldsymbol{\varepsilon}_{\delta}^{(n)}+\mu \frac{\mathbf{L}_{i}^{H}}{\left\|\mathbf{L}_{i}\right\|_{2}^{2}}\left(\mathbf{E}_{\mathbf{i}}^{\mathbf{s}}-\mathbf{L}_{i} \cdot \boldsymbol{\varepsilon}_{\delta}^{(n)}\right) .
$$

The algorithm is terminated by looking at the evolution of the residual $r^{(n)}$ :

$$
r^{(n)}=\left\|\mathbf{E}^{s}-\mathbf{L} \cdot \boldsymbol{\varepsilon}_{\delta}^{(n)}\right\|_{2}
$$

For every $n>1, r^{(n)}$ is compared to $r^{(n-1)}$. If the difference is less than $5 \%$ of the maximum difference ever observed, the algorithm is terminated and the corresponding solution $\boldsymbol{\varepsilon}_{\delta}^{(n)}$ is returned.

This basic method can be further improved with the inclusion of a simple physical bound on the solution returned. Using the standard notation $\varepsilon=\varepsilon_{0} \varepsilon_{r}(1-j \tan \delta)$, at every ART iteration we force the solution to have positive real part and negative imaginary part, which is valid in free space or for a loss-less host medium. This physical bound is hardimposed on the solution by flipping the sign of the 
elements that do not respect it. This can have detrimental effects on the solution unless the effect of this modification is not smoothed. Therefore, the multiplier $\mu$ in (5) is empirically chosen so as to achieve meaningful reconstruction. The multiplier acts as a regularizing parameter. A large value $(\mu \approx 1)$ makes the solution sharper but could introduce noise, while a small $\mu$ tends to smooth the final image.

2.3. Experimental Setup. We designed, built, and programmed an automated antenna-positioning system, shown in Figure 1, which moves the antennas, samples the electric field, and stores the measurement data for later analysis.

Precise positioning of the antennas is paramount to achieve good quality images. The two antennas are moved along circles of different radii. Angular positioning with an error significantly smaller than $1^{\circ}$ is achieved by using stepper motors in microstepping mode, and by using a pulley-belt system with a gear ratio of $4: 1$. The system is controlled by a LabVIEW application, which coordinates the movement of the antennas with the RF measurements.

Simple half-wavelength dipoles are used in the experiments instead of the electrically short dipoles of the forward model. This choice was made because half-wavelength dipoles are more readily available, provide a much higher radiation resistance, and have a lower reflection coefficient to allow for a more efficient use of the input power. The antennas were operated at $3.16 \mathrm{GHz}\left(\lambda_{0} \approx 9.5 \mathrm{~cm}\right)$, with a transmit power of $20 \mathrm{dBmW}$. The frequency has been chosen to allow a proper scaling of the problem and has then been finely tuned to operate the antennas with minimum mismatch. The antennas were moved around the targets and the field was sampled at each position. This approach has the advantages of reducing the complexity and the cost of the measurement system, and of limiting the mutual coupling between the antennas.

A vector network analyzer (HP 8753ES) measured the scattering parameter $S_{21}$. For each measurement 16 samples were taken and averaged to reduce additive white Gaussian noise effects. The measurements were taken in an anechoic room to further reduce undesired reflections and to mitigate external RF sources in the same frequency range. $\mathrm{TM}^{z}$ polarization (i.e., vertical, perpendicular to the floor plane) was used.

2.4. Test on Simulated Data. To compare the performance of the ART inversion against the TSVD algorithm used in [2-4], we chose a sample test case. This test case has been simulated using the method of moments to obtain the scattered electric field. The data has then been used to reconstruct the shape of the target using TSVD, ART, and ART with the introduction of the physical bounds.

The simulation conditions are the same that have later been tested in the laboratory, and they are shown in Figure 2. Operating at $3.16 \mathrm{GHz}$, we attempt to obtain images of a simple target, consisting of a short section of a PVC pipe: its height is $4.8 \mathrm{~cm}\left(\approx 0.51 \lambda_{0}\right)$, its outer diameter is $4.8 \mathrm{~cm}$ $\left(\approx 0.51 \lambda_{0}\right)$, its inner diameter is $4.4 \mathrm{~cm}\left(\approx 0.46 \lambda_{0}\right)$, and therefore the thickness of the PVC is $4 \mathrm{~mm}\left(\approx 0.04 \lambda_{0}\right)$; the

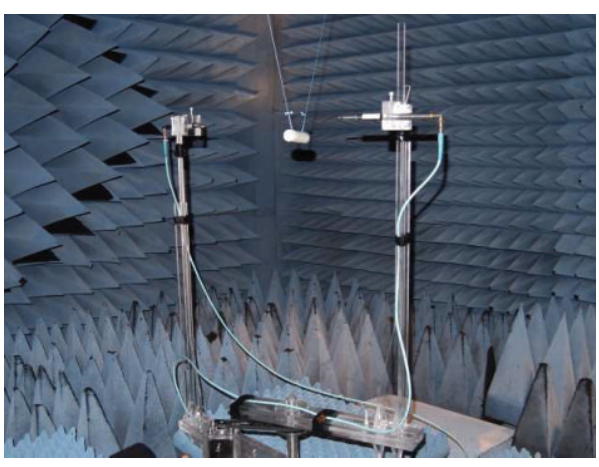

FIgURE 1: Experimental setup. The TX is on the left, the RX is on the right, and a target is visible in the top center, hanging from the ceiling.

dielectric constant of PVC is estimated to be $\varepsilon_{r}=2.5$ and its conductivity is negligible $(\tan (\delta)=0.004$ at $3 \mathrm{GHz})$. The target and the antenna positions are shown in Figure 2. The object is illuminated from 11 positions along a circumference of radius $38.4 \mathrm{~cm}$, uniformly spaced between $0^{\circ}$ and $280^{\circ}$. The electric field is sampled at 20 locations along a circumference of radius $15.4 \mathrm{~cm}$, uniformly spaced between $0^{\circ}$ and $360^{\circ}$.

All images are obtained considering a square window of sides equal to $20 \mathrm{~cm}$, centered at the axis of rotation of the antennas, and divided into 101 pixels per side. Each pixel is therefore $2 \mathrm{~mm}$ (or approximately $\lambda_{0} / 47$ ). This leads to a problem with $101 \times 101=10201$ unknowns which must be reconstructed from 220 measurements. The conditioning number $\kappa(\mathbf{L})$ turns out to be approximately equal to $1.7 \times$ $10^{5}$. The problem is therefore largely underdetermined and illconditioned.

We reconstructed the absolute value of the dielectric permittivity contrast function, $\left|\boldsymbol{\varepsilon}_{\delta}\right|$. Figure $3(\mathrm{a})$ shows the reconstruction obtained using TSVD, in its MATLAB implementation, which computes all of the singular values (even those that will be discarded later). The image is obtained by retaining 74 out of 220 singular values. This value has been automatically determined by choosing the solution representing the best compromise between the norm of the solution $\left\|\varepsilon_{\delta}\right\|_{2}$ and the norm of the residual (6). This procedure is commonly referred to as the analysis of the L curve [15]. The image thus obtained barely allows to understand the shape of the object, and it is noisy and blurry.

Figure 3(b) shows the reconstruction obtained using the ART method in its original, unmodified version. Reconstruction is comparable to TSVD but the shape of the target cannot be inferred correctly. The algorithm is stopped after 4 iterations, when the residual $r$ changes by less than $5 \%$, as explained in the previous section. The value of $\mu$ has been empirically chosen to be 1 .

The best image is obtained by applying physical bounds to the ART method, as shown in Figure 3(c). The image has been obtained with the same value of $\mu$, and is obtained after 8 iterations (less than 5\% residual changes). The image is clearly superior to both TSVD and ART: the thickness of the pipe is better estimated and the image is by far less 


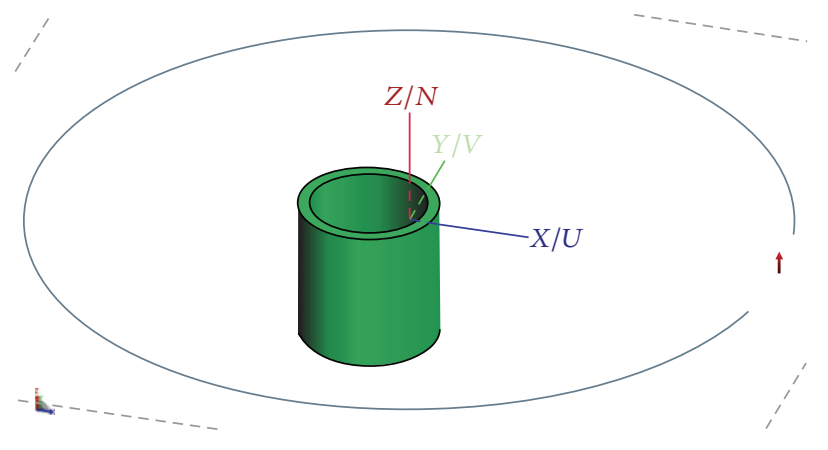

(a)

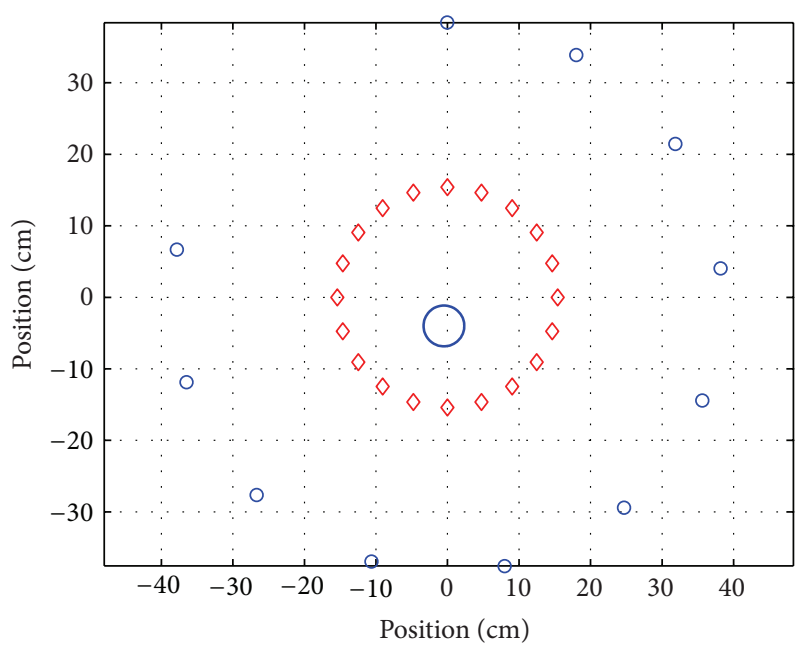

O TX

$\diamond \mathrm{RX}$

— Target

FIgURE 2: 3D model of the PVC pipe (a) and locations of the antennas used in the measurement (b).

noisy. Therefore we observe that the sole introduction of the physical bound not only has the benefit of restricting the space where a solution is searched, but it also reduces noise and increases the resolution achievable by the inversion, showing sharpening properties.

Another comment can be made about the computational resources needed by TSVD and ART. TSVD is far more demanding than ART: the computation of the singular value decomposition requires time and a lot of memory. On a laptop computer equipped with $1.5 \mathrm{~GB}$ of memory the inversion cannot be completed because the software runs out of memory. On the other hand, ART is affected by the fact that it nests two iterations: the outer one updates the solution, the inner one generates a solution by scanning all the rows of the matrix $\mathbf{L}$. These nested loops are not efficiently executed in MATLAB, resulting in a computational time required which is comparable to TSVD. The memory requirement is however extremely smaller, and a proper efficient implementation of the code in a compiled language such as $\mathrm{C}$ would speed up the algorithm significantly.

In conclusion, we observe that the reconstruction obtained using ART with the introduction of the physical bound is superior to TSVD, and it is chosen as the only method used for the inversion in the following.

\section{Results and Discussion}

In this section we present reconstruction from measurements obtained using the system described above.

3.1. Empty PVC Pipe. For the first measurement result, we replicated the conditions of the simulation shown in Figure 3.
Figure 4 shows the reconstruction results. To show the effect of the multiplier $\mu$ on the final solution, we reported two cases, with $\mu=0.8$ and $\mu=1.4$, respectively. Reconstruction is extremely satisfactory, showing minimal differences when compared to the simulation result. For both values of $\mu$, the algorithm finished in 7 iterations.

3.2. Two Nylon Rods. The second case study involves imaging two nylon rods (diameter equal to $2.5 \mathrm{~cm}, \varepsilon_{r} \approx 2.84$, and $\tan \delta \approx 0.012$ at $3 \mathrm{GHz}$ ). The centers of the two rods are separated by $3 \mathrm{~cm}\left(\approx \lambda_{0} / 3\right)$; that is, the sides of the rods are separated by $0.5 \mathrm{~cm}\left(\approx \lambda_{0} / 20\right)$. The result is shown in Figure 5 . For this measurement, due to mechanical reasons, the transmitting antenna was moving along a circumference of radius $38.2 \mathrm{~cm}$ and the receiving antennas along a circumference of radius $15.7 \mathrm{~cm}$.

Reconstruction is again successful although the estimation of the size and separation of the two objects is not straightforward. To estimate size and separation of the objects we adopted a standard metric. The images are normalized to their maximum, and the objects are considered present whenever the absolute value of the reconstructed permittivity is greater than 0.5. Using this metric, the objects reconstructed in Figure 5(a) appear as having a diameter of $2.8 \mathrm{~cm}$ (average value, due to nonperfect circular shapes), which yields an error of approximately $12 \%$. The separation between the objects, instead, appears to be equal to $0.6 \mathrm{~cm}$, an error of $20 \%$. These estimations are also affected by the pixel size, which introduces an uncertainty of $\pm 2 \mathrm{~mm}$.

As expected, a smaller $\mu$ makes the objects appear larger, and also closer in space. Conversely, a larger $\mu$ underestimates the size of the objects, which also appear more separated than they are in reality. The choice of $\mu$ is therefore very 


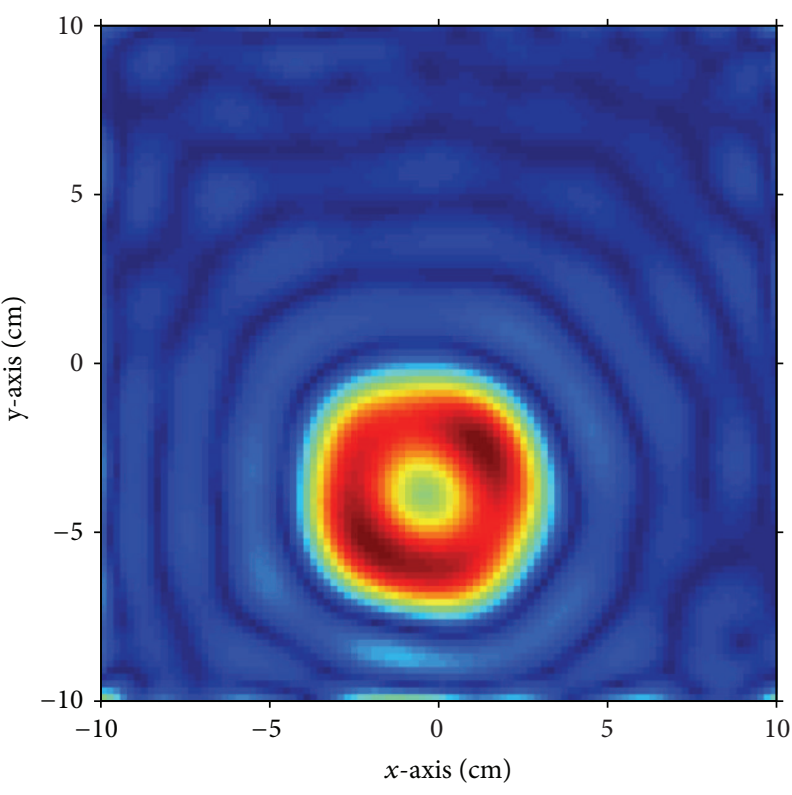

(a) TSVD

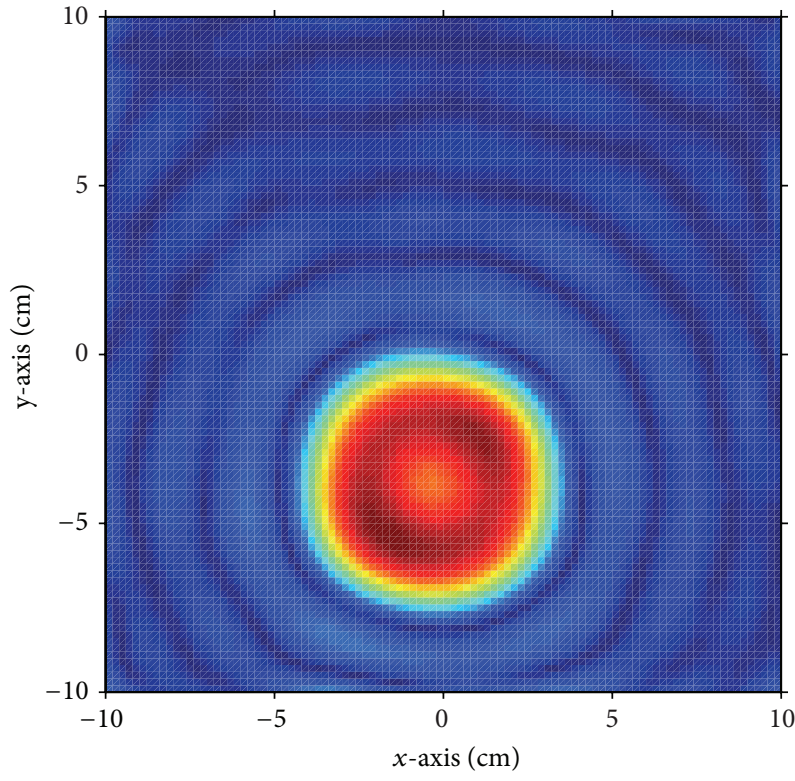

(b) ART

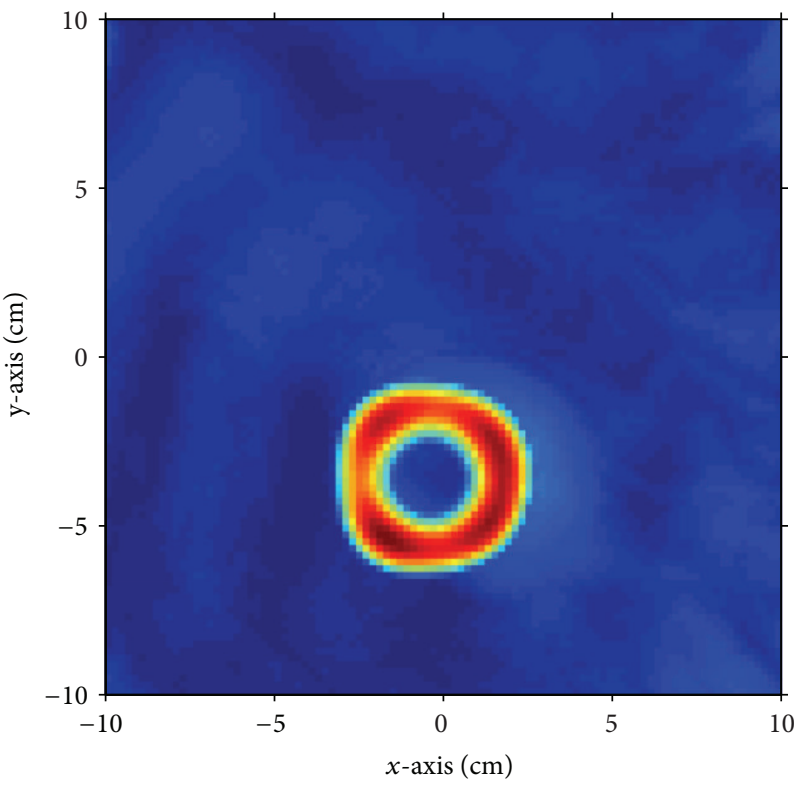

(c) ART with physical bounds

FIgURE 3: The figure shows reconstruction from simulated scattered electric fields, for an empty PVC pipe. TSVD (a) offers reconstruction very similar to ART (b), but the best image is obtained by using ART with the introduction of physical bounds (c). Both (b) and (c) have been obtained with $\mu=1$. All images have been normalized to their maximum since quantitative reconstruction is not of interest.

important and a certain strategy for choosing its value should be researched in the future.

3.3. Metallic and Plastic Objects. The third test case serves to validate the ability of the imaging system to distinguish between metallic and plastic cylinders. The enforcement of the physical bounds guarantees that the real part of the image is positive and the imaginary part is negative. Separating real and imaginary parts should provide a way to separate conducting versus nonconducting objects.
The following test has been carried out using 16 transmitters rotating around a circle of radius equal to $43.2 \mathrm{~cm}$ and 40 receivers whose radius of rotation was $22 \mathrm{~cm}$.

Two targets are present in the area under investigation. The first target is a copper cylinder, of diameter $2.5 \mathrm{~cm}$ and $4 \mathrm{~cm}$ tall. The cylinder is hollow and its thickness is equal to $1 \mathrm{~mm}$. The object is placed in the lower portion of the area under reconstruction. The second target is a plastic cylinder (acrylic glass, $\varepsilon_{r} \approx 2-3.5$ at $3 \mathrm{GHz}$ ), with diameter $2.5 \mathrm{~cm}$ and height $5.1 \mathrm{~cm}$. This cylinder is also hollow, with its side having 


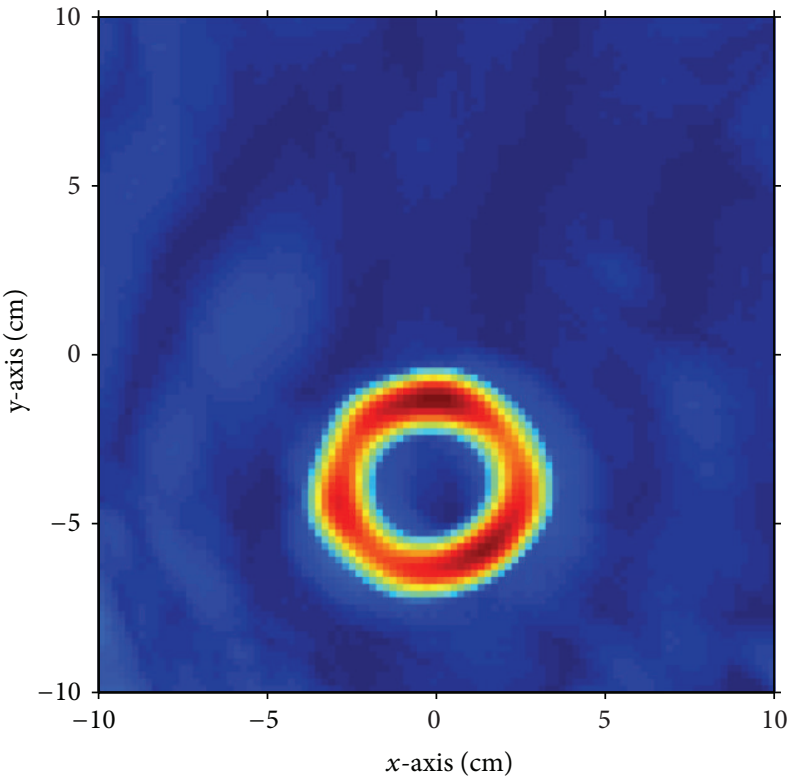

(a)

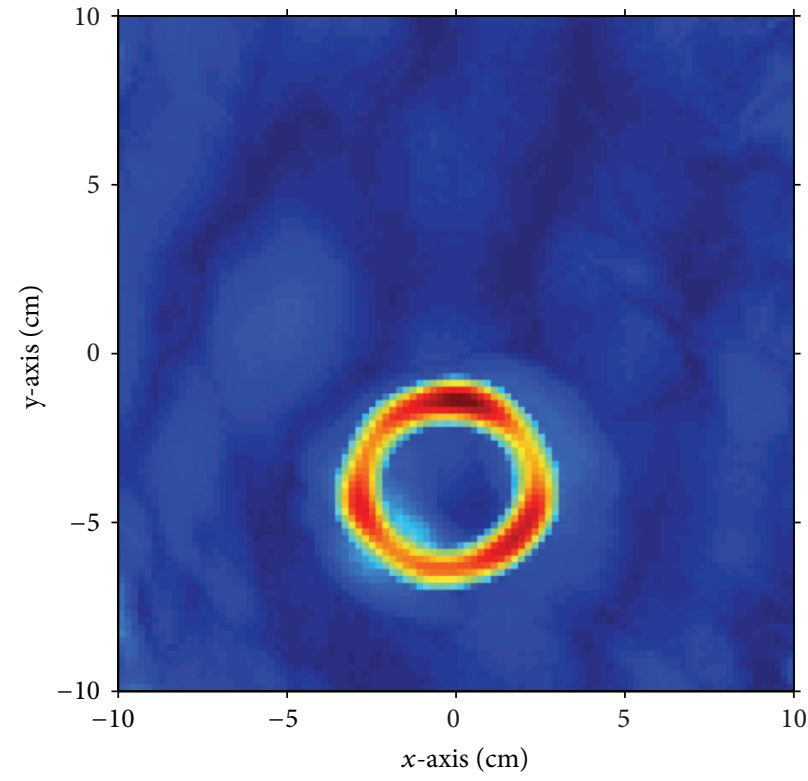

(b)

FIGURE 4: Reconstruction of PVC pipe from measurements, using ART with the enforcement of the physical bounds. The figure shows the reconstruction differences when using $\mu=0.8$ (a) and $\mu=1.4$ (b).

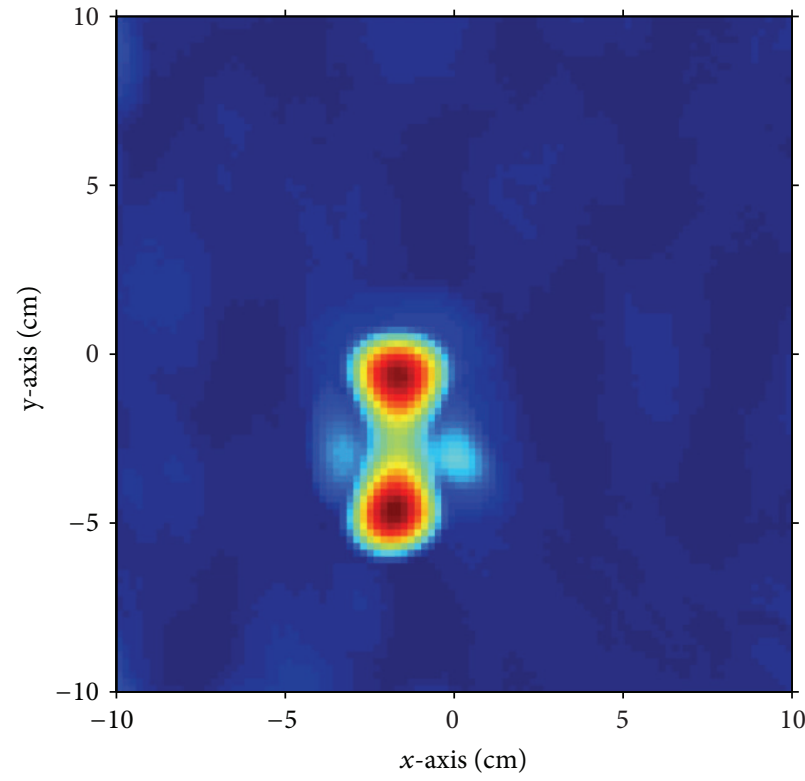

(a)

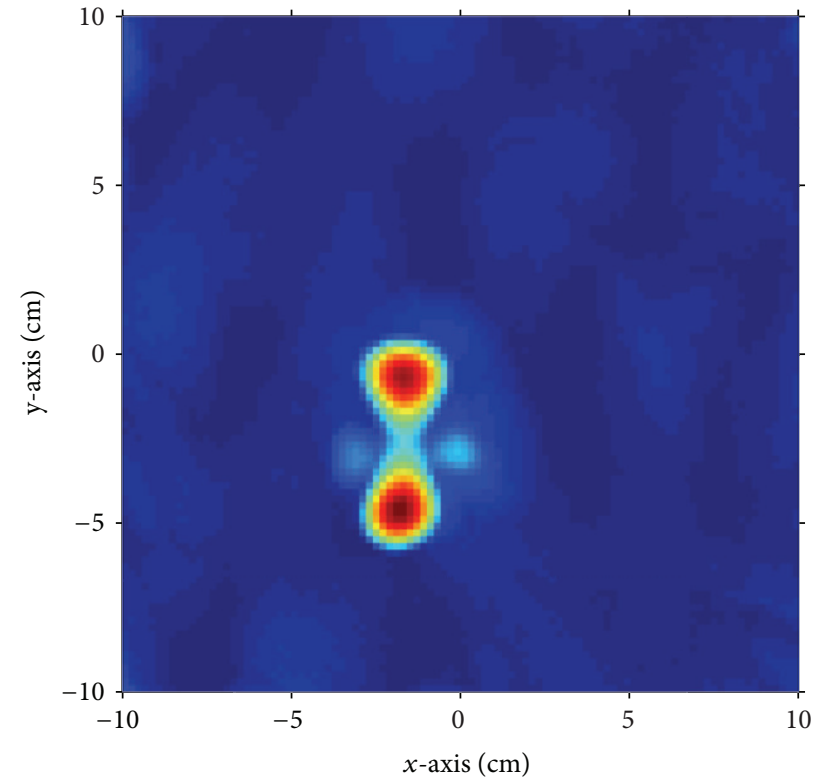

(b)

FIgURE 5: Reconstruction of two nylon rods. The figure shows the reconstruction differences when using $\mu=1$ (a) and $\mu=1.4$ (b).

a thickness of $6 \mathrm{~mm}$. This second cylinder is located in the upper left portion of the area under reconstruction.

The reconstruction obtained using ART with the enforcement of the physical bounds is shown in Figure 6. The absolute value of the dielectric permittivity distribution shows a prominent object at the location of the metallic target. This is normal since metal represents a much stronger scatterer than plastic. However, when the image is split between its real and imaginary parts, the plastic and metallic objects are reconstructed correctly. The imaginary part shows a small but not zero conductivity for the plastic cylinder, while the real part is instead nonzero only for the plastic target.

To evaluate the effect of the physical bound, we can compare the reconstruction shown in Figure 6 against the one obtained by the same ART code, with the same $\mu$, but without the enforcement of the physical bound. The result is 


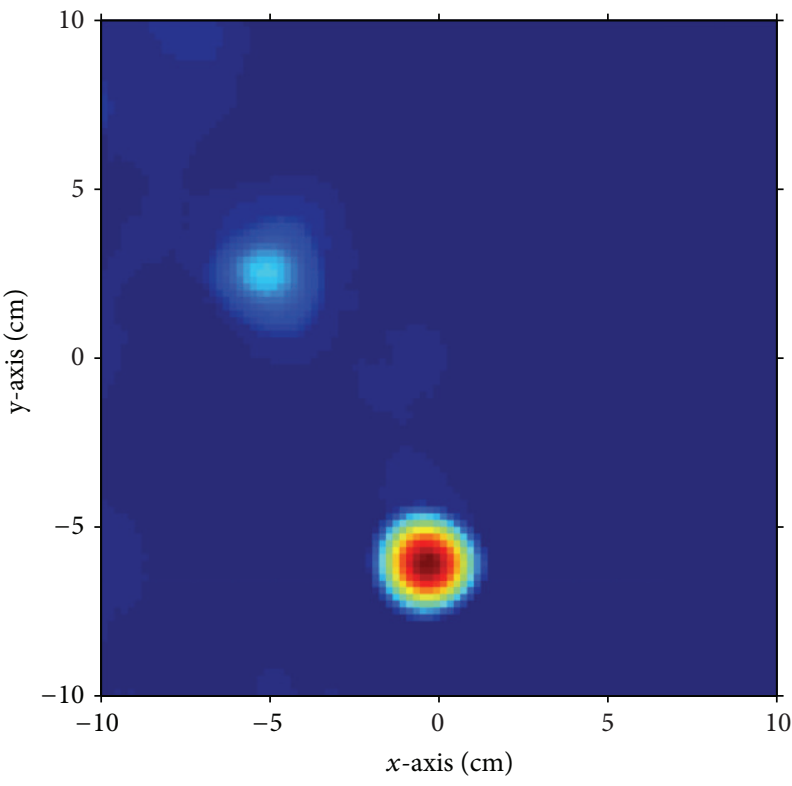

(a)

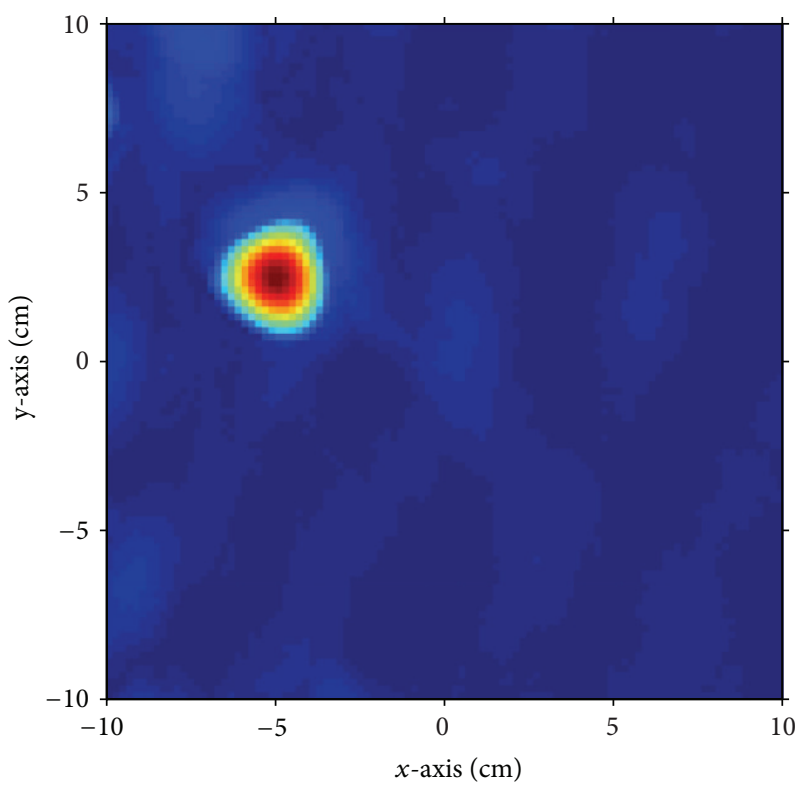

(b)

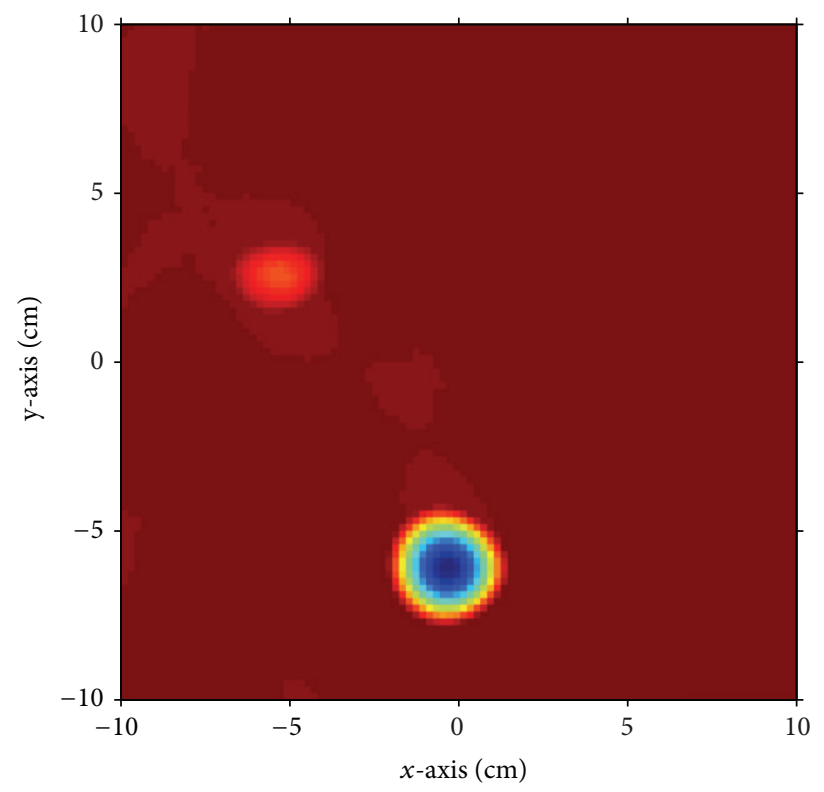

(c)

FIGURE 6: ART method with enforcement of physical bounds. (a) shows $\left|\boldsymbol{\varepsilon}_{\delta}\right|$, (b) shows $\operatorname{Re}\left(\boldsymbol{\varepsilon}_{\delta}\right)$, and (c) shows $\operatorname{Im}\left(\boldsymbol{\varepsilon}_{\delta}\right)$. All images are obtained with $\mu=0.2$ and are normalized.

shown in Figure 7. The image is still reconstructed somewhat correctly: the real and imaginary parts show metallic and plastic objects in the right location. However, the image is much noisier, and reconstruction is not as efficient. This shows that the introduction of the forced physical bound does not necessarily correct a wrong reconstruction, but it is extremely effective in reducing the noise in the final image.

\section{Conclusions}

In this paper, we described a measurement system used to validate the forward model of radio frequency tomography and an inversion method based on the algebraic reconstruction technique. The original forward model of RF Tomography has been modified with the introduction of a dyadic Green's function for free space, due to the conditions in which the experiments took place. This also allowed us to test the flexibility of RF Tomography to operate in scenarios different from the one for which it was originally intended.

Regarding the inversion algorithm, we developed an inversion scheme to reconstruct shape and location of the targets, without attempting to estimate their exact permittivity values. An algorithm based on the Algebraic Reconstruction Technique, augmented with the enforcement of a physical 


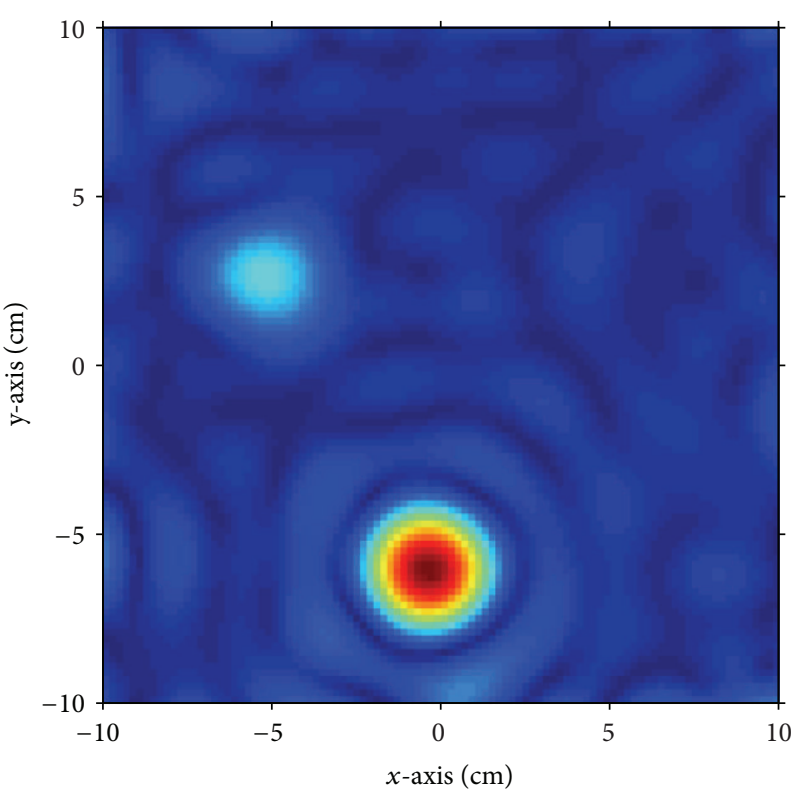

(a)

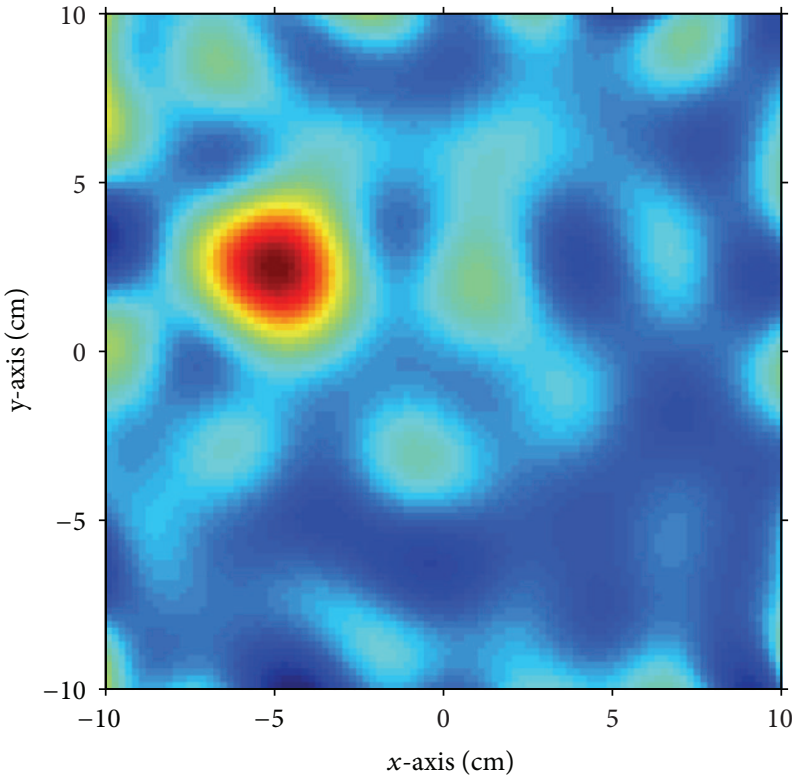

(b)

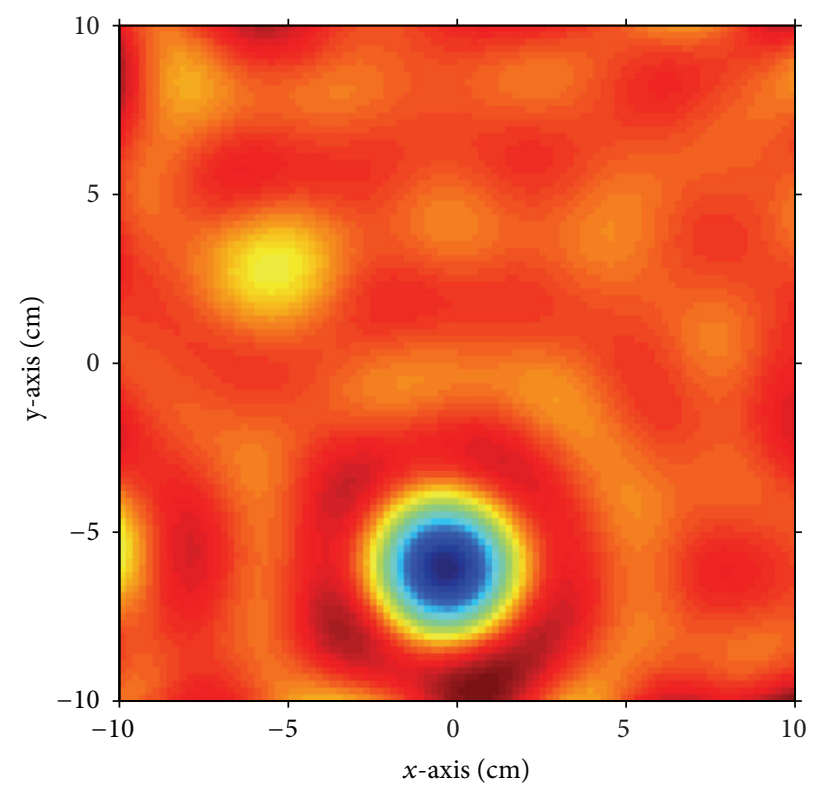

(c)

FIGURE 7: Standard ART method. (a) shows $\left|\boldsymbol{\varepsilon}_{\delta}\right|$, (b) shows $\operatorname{Re}\left(\boldsymbol{\varepsilon}_{\delta}\right)$, and (c) shows $\operatorname{Im}\left(\boldsymbol{\varepsilon}_{\delta}\right)$. All images are obtained with $\mu=0.2$ and are normalized.

bound, satisfied our requirements. The developed algorithm proved to be at least as quick as TSVD, with a much more limited memory usage, and could provide actionable results from a very limited number of measurements. The reconstruction is superior to the one obtained by TSVD both in terms of quality and of computational resources required.

The results obtained through imaging of dielectric targets are encouraging. Despite the weak scatterers used, reconstruction has been successful. The resolution of the system is good and able to correctly reconstruct objects which are separated by a few millimeters. Metal and plastic objects can be reconstructed separately, obtaining images with very little noise, thanks to the physical bound imposed on ART. The problem of resolution provides motivation for further research, as does the problem of finding a value of $\mu$ nonempirically. In addition, we intend to study more complex geometries, both in terms of targets and environment (halfspace, through wall, etc.).

\section{Acknowledgments}

This research was supported by AFRL through Grant FA955012-1-0174, by RNET and AFRL through Grant FA8659010D-1750, and the UIC Chancellor Undergraduate Research 
Award. The authors would like to thank M. Stephens and L. Patel of UIC.

\section{References}

[1] M. C. Wicks, "Rf tomography with application to ground penetrating radar," in Proceedings of the IEEE 41st Asilomar Conference on Signals, Systems and Computers (ACSSC '07), pp. 2017-2022, 2007.

[2] L. L. Monte, D. Erricolo, F. Soldovieri, and M. Wicks, "Radio frequency tomography for tunnel detection," IEEE Transactions on Geoscience and Remote Sensing, vol. 48, no. 3, pp. 1128-1137, 2010.

[3] L. L. Monte, D. Erricolo, F. Soldovieri, and M. C. Wicks, "RF tomography for below-ground imaging of extended areas and close-in sensing," IEEE Geoscience and Remote Sensing Letters, vol. 7, no. 3, pp. 496-500, 2010.

[4] L. L. Monte, F. Soldovieri, D. Erricolo, and M. C. Wicks, "Imaging below irregular terrain using RF tomography," IEEE Transactions on Geoscience and Remote Sensing, vol. 50, no. 9, pp. 3364-3373, 2012.

[5] F. Soldovieri, L. L. Monte, and D. Erricolo, "Tunnel detection and localisation via multi-monostatic radio frequency tomography using magnetic sources ," IET Radar, Sonar \& Navigation, vol. 6 , no. 9, article 834, 2012.

[6] L. L. Monte, F. Soldovieri, D. Erricolo, A. Giannopoulos, and M. C. Wicks, "A comprehensive forward model for imaging under irregular terrain using RF tomography," International Journal of Antennas and Propagation, vol. 2012, Article ID 735414, 14 pages, 2012.

[7] W. C. Chew, Waves and Fields in Inhomogeneous Media, IEEE Press, New York, NY, USA, 1995.

[8] L. Tsang, J. A. Kong, and K. H. Ding, Scattering of Electromagnetic Waves, Theories and Applications, Wiley-Interscience, New York, NY, USA, 2004.

[9] L. L. Monte, J. T. Parker, R. Vela, and C. Baker, "A calibration procedure for ground-based RF tomography," in Proceedings of the IEEE Radar Conference (RADAR '12), pp. 635-640, Atlanta, Ga, USA, May 2012.

[10] S. Nishikata, V. Picco, T. Negishi, and D. Erricolo, "Imaging of dielectric targets using rf tomography," in Proceedings of the 2012 IEEE International Symposium on Antennas and Propagation and USNC/URSI National Radio Science Meeting, 2012.

[11] J. T. Parker, M. Ferrara, J. Bracken, and B. Himed, "Preliminary experimental results for RF tomography using distributed sensing," in Proceedings of the 12th International Conference on Electromagnetics in Advanced Applications (ICEAA '10), pp. 549-552, September 2010.

[12] J.-M. Geffrin, P. C. Chaumet, C. Eyraud, K. Belkebir, and P. Sabouroux, "Electromagnetic three-dimensional reconstruction of targets from free space experimental data," Applied Physics Letters, vol. 92, no. 19, Article ID 194103, 2008.

[13] C. Yu, M. Yuan, J. Stang et al., "Active microwave imaging II: 3-D system prototype and image reconstruction from experimental data," IEEE Transactions on Microwave Theory and Techniques, vol. 56, no. 4, pp. 991-1000, 2008.

[14] C. Gilmore, P. Mojabi, A. Zakaria et al., "A wideband microwave tomography system with a novel frequency selection procedure," IEEE Transactions on Biomedical Engineering, vol. 57, no. 4, pp. 894-904, 2010.

[15] P. C. Hansen, "Analysis of discrete ill-posed problems by means of the L-curve," SIAM Review, vol. 34, no. 4, pp. 561-580, 1992. 

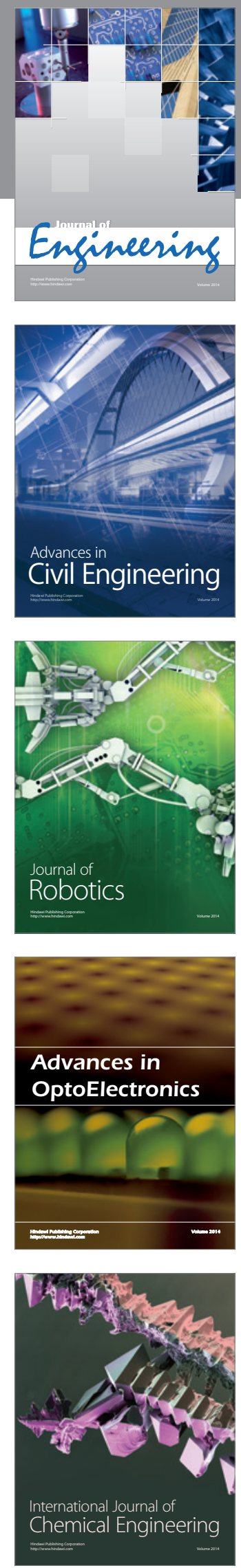

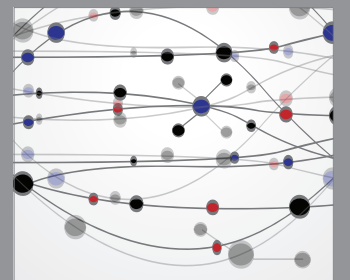

The Scientific World Journal
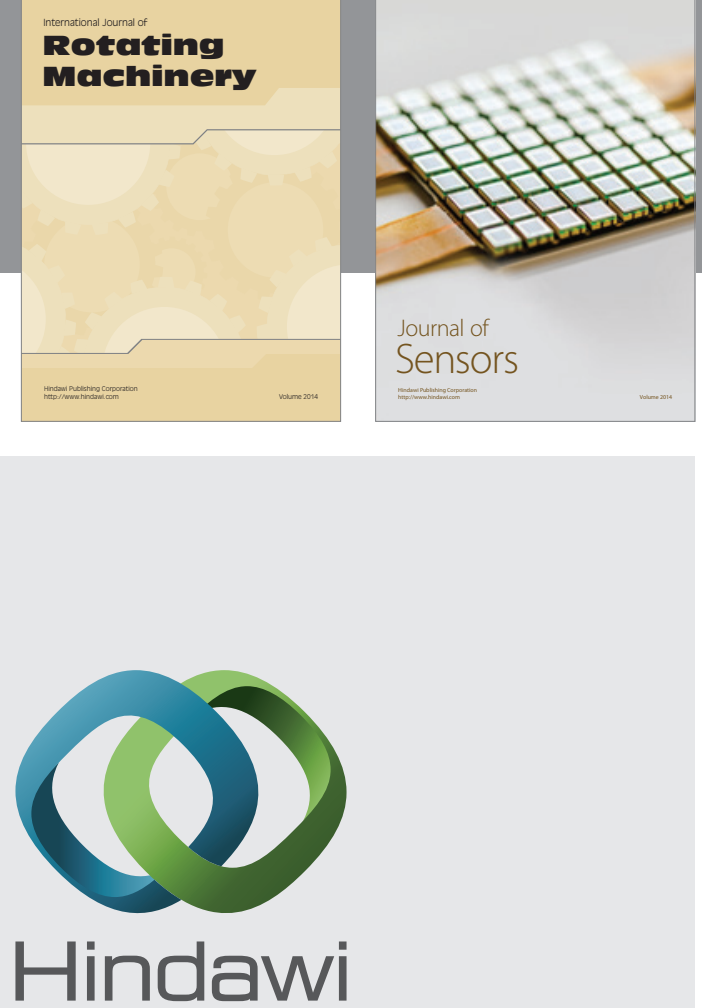

Submit your manuscripts at http://www.hindawi.com
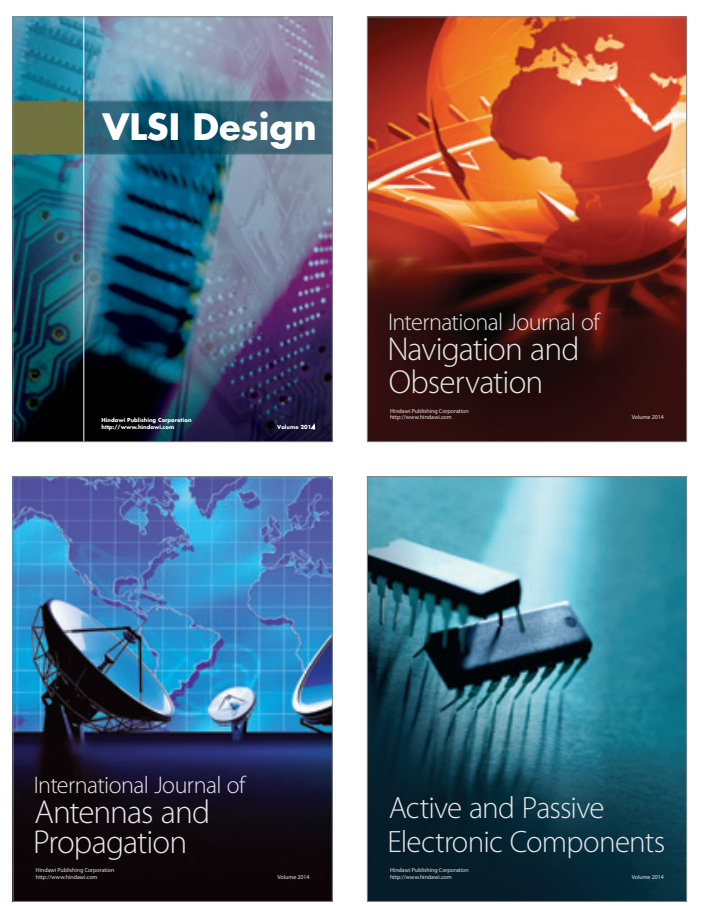
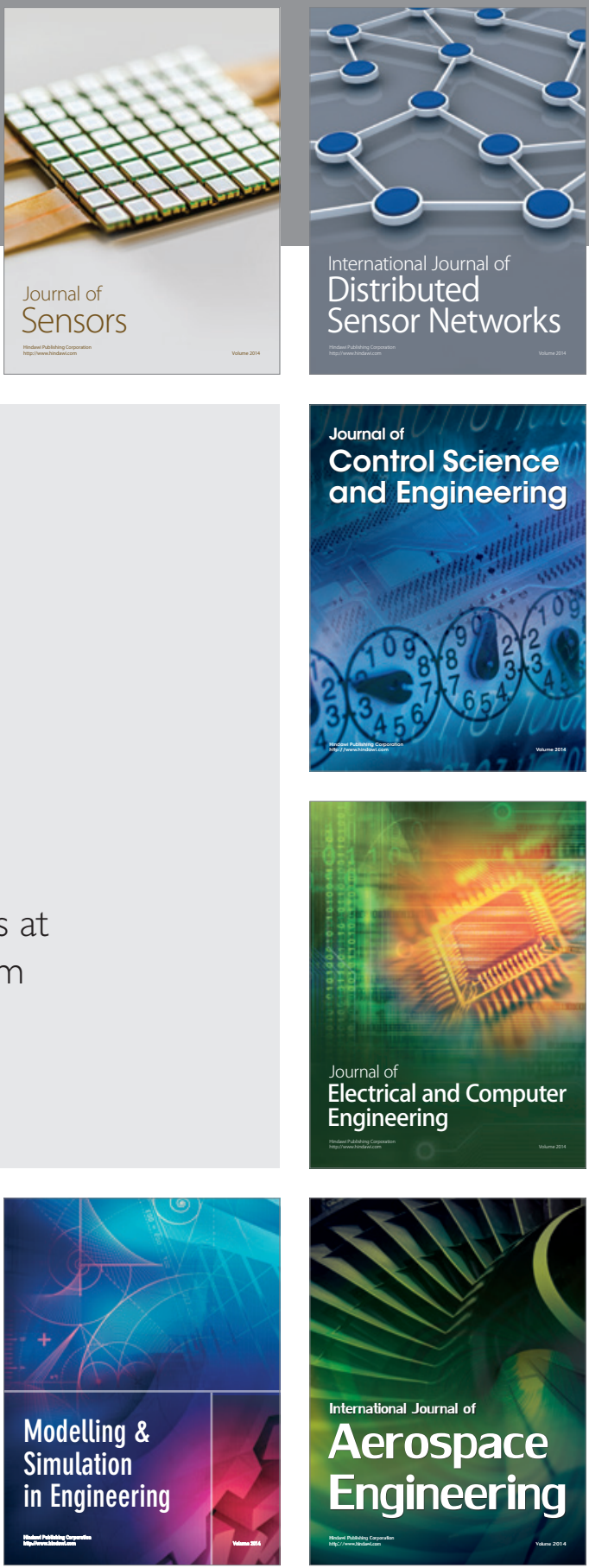

Journal of

Control Science

and Engineering
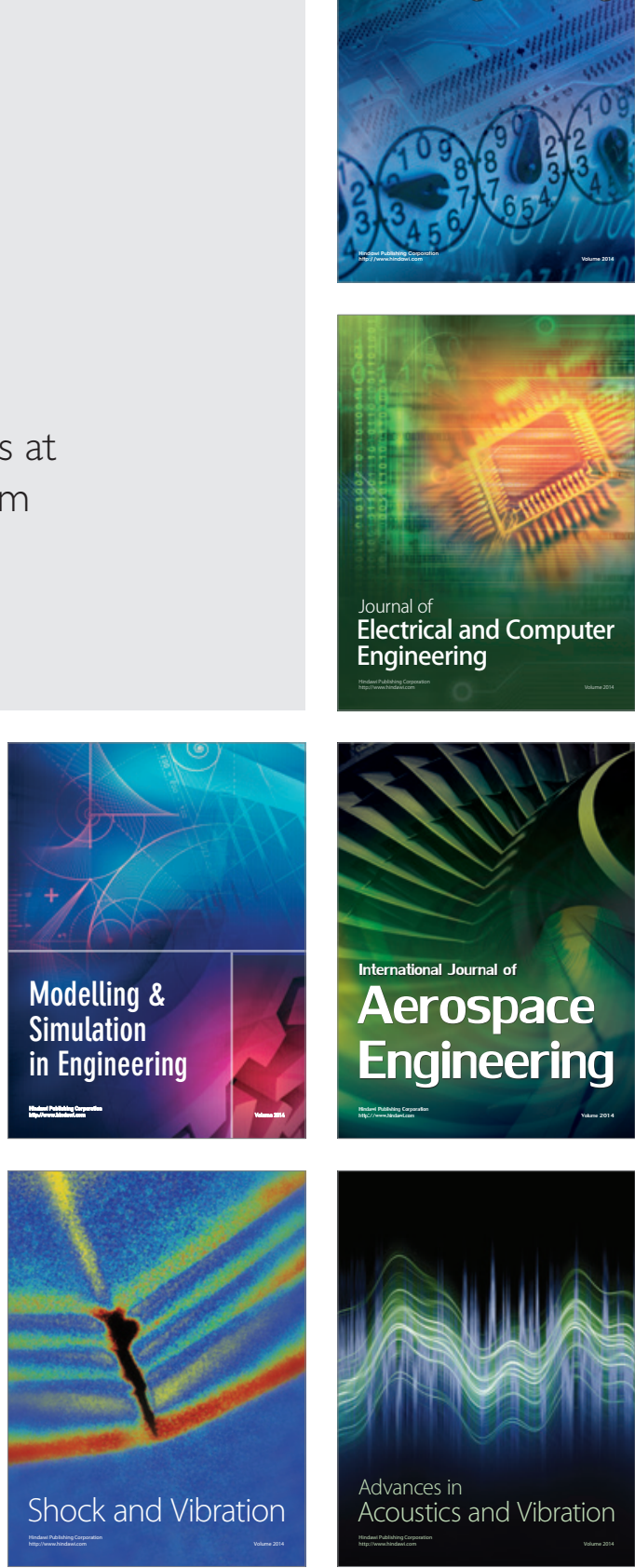